\title{
HELIUM FLASHES THROUGH THE NCO REACTION
}

\author{
K. Arai and K. Kaminisi \\ Department of Physics, Kumamoto University, Kumamoto 860
} M. Hashimoto

Max Planck Institut für Astrophysik, Garching bei München D-8046 and $K$. Nomoto

Department of Earth Science and Astronomy, University of Tokyo

Meguro-ku, Tokyo 153

\section{Introduction}

It is generally accepted that the helium flash occurs when the $3 \alpha$ reaction commences in the degenerate helium core of low mass stars. In this core, original CNO isotopes have been converted into ${ }^{14} \mathrm{~N}$ and the electron Fermi energy becomes large enough to approach the threshold energy for e-capture on ${ }^{14} \mathrm{~N}$. Hence Kaminisi et al. (1975) have pointed out that in these circumstances the ${ }^{14} \mathrm{~N}\left(\mathrm{e}^{-}, \nu\right)^{14} \mathrm{C}(\alpha, \gamma){ }^{18} \mathrm{O}$ (NCO) reaction may play an important role for igniting the helium flash.

We, therefore, examine the effects of the Nco reaction on the evolution of low mass stars. A key ingredient of the NCo reaction is that the density reaches the threshold for e-capture $\left(\rho_{\mathrm{th}} \sim 10^{6} \mathrm{~g} \mathrm{~cm}^{-3}\right)$. Evolutionary sequences are presented for the cases of accreting helium white dwarfs (Hashimoto et al. 1986) and a $0.7 \mathrm{M}_{\odot}$, Population II star ascending the giant branch.

\section{Evolution of accreting helium white dwarfs}

An initial model has the mass $M=0.3 \mathrm{M}$ and the chemical abundances $x\left({ }^{4} \mathrm{He}\right)=0.9879$ and $x\left({ }^{14} \mathrm{~N}\right)=0.0121$. In three cases, $A, B$ and $C$,

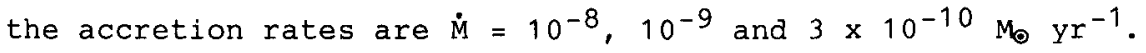

The evolutionary paths of the central density and temperature are plotted by the solid lines in Figure 1 for cases A-C. The dashed lines show the paths for the same accretion rates as for cases A-C but with the NCO reaction switched off. The dotted lines denote the ignition curves for the $3 \alpha$ and NCO reactions. Note that the NCO reaction dominates over the $3 \alpha$ reaction to heat up the core. The inclusion of the Nco reaction leads to the ignition of the helium flash at considerably lower density.

In case $A$, once $\rho_{C}$ reaches $\rho_{\text {th }}$, produced ${ }^{14} \mathrm{C}$ quickly burns to ${ }^{18} \mathrm{O}$. The core halts further contraction. The helium flash is initiated by the NCO reaction. In cases $B$ and $C$, the condition $\rho_{C}>\rho_{\text {th }}$ is realized. Then ${ }^{14} \mathrm{~N}$ is completely converted into ${ }^{14} \mathrm{C}$ in the central region. However, ${ }^{14} \mathrm{C}$ has not yet been processed into $18{ }_{\mathrm{O}}$, because $\mathrm{T}_{\mathrm{C}}$ is too low for the $\alpha$-capture to occur. The central density continues to increase until the helium flash is triggered by ${ }^{14} \mathrm{C}$ burning. 
3. Evolution of a low mass star ascending the giant branch

Referring to a specified sequence of a $0.7 \mathrm{M}_{\odot}$ star presented by Sweigart and Gross (1978), we calculate the red giant sequence from the subgiant branch to the onset of the helium flash. The initial composition is $x\left({ }^{4} \mathrm{He}\right)=0.999$ and $x\left({ }^{14} \mathrm{~N}\right)=0.001$.

The evolutionary track followed by the center and the temperature profiles against density are shown by the solid lines in Figure 2. The numerals attached to the lines denote the time before the onset of the flash in units of $10^{6} \mathrm{yr}$. The center does not reach the Nco ignition curve before the $3 \alpha$ reaction ignites at the site of the maximum temperature. The NCO reaction does not change the existing evolutionary models, as was pointed out by spulak (1980), except that a considerable amount of ${ }^{18} \mathrm{O}$ is produced in the central region.

\section{Concluding remarks}

It is shown that the original CNO isotopes in the core of a low mass star are converted into 180 during the helium flash. We note that the NCO and the subsequent ${ }^{18} \mathrm{O}(\alpha, \gamma)^{22} \mathrm{Ne}(\alpha, n)^{25} \mathrm{Mg}$ reactions can be a prolific source of neutrons for the synthesis of s-process elements.

\section{References}

Hashimoto, M., et al. 1986, Ap. J., 307, 687.

Kaminisi, K., et al. 1975, Prog. Theor. Phys., 53, 1855.

Spulak, R. G., Jr. 1980, Ap. J., 235, 565.

Sweigart, A. V., and Gross, P. G. 1978, Ap. J. Suppl., 36, 405.

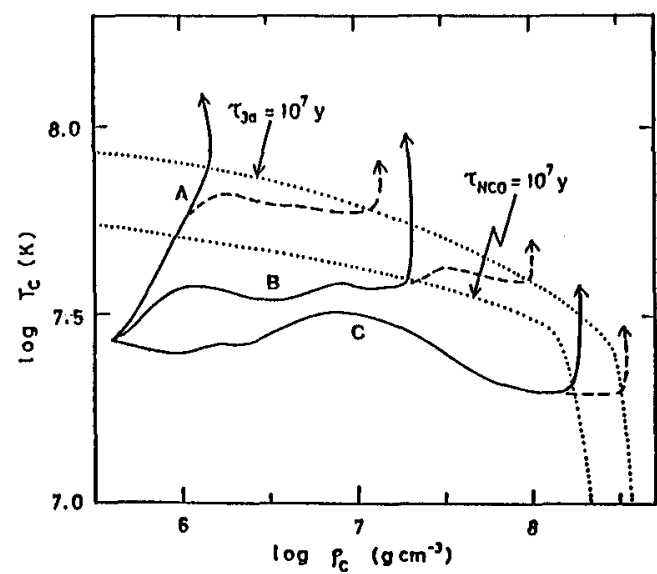

Fig. 1. Evolutionary tracks of accreting helium white dwarfs.

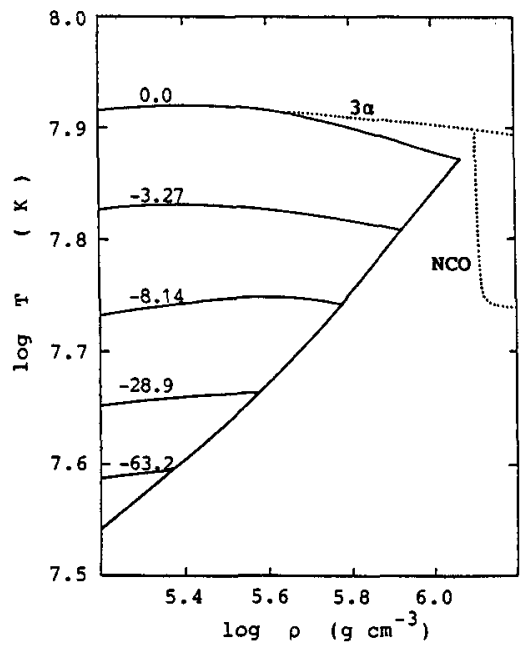

Fig. 2. Evolutionary track of a 0.7 Mo star. 